\title{
Mefloquine exerts anticancer activity in prostate cancer cells via ROS-mediated modulation of Akt, ERK, JNK and AMPK signaling
}

\author{
KUN-HUANG YAN ${ }^{1 *}$, CHIH-JUNG YAO ${ }^{2-4 *}$, CHI-HAO HSIAO ${ }^{1}$, KE-HSUN LIN ${ }^{1}$, \\ YUNG-WEI LIN ${ }^{1}$, YU-CHING WEN ${ }^{1}$, CHUNG-CHI LIU ${ }^{1}$, MING-DE YAN ${ }^{5}$, \\ SHUANG-EN CHUANG ${ }^{6}$, GI-MING LAI ${ }^{2-4,6,7^{*}}$ and LIANG-MING LEE ${ }^{1,8^{*}}$
}

\begin{abstract}
${ }^{1}$ Department of Urology, Wan Fang Hospital, Taipei Medical University; ${ }^{2}$ Department of Internal Medicine, School of Medicine, College of Medicine; ${ }^{3}$ Cancer Center, Wan Fang Hospital; ${ }^{4}$ Center of Excellence for Cancer Research;

${ }^{5}$ Division of Gastroenterology, Department of Internal Medicine, Wan Fang Hospital, Taipei Medical University,

Taipei 11696; ${ }^{6}$ National Institutes of Cancer Research, National Health Research Institutes, Miaoli 35053;

${ }^{7}$ Division of Hematology and Medical Oncology, Department of Internal Medicine, Wan Fang Hospital;

${ }^{8}$ Department of Urology, School of Medicine, College of Medicine, Taipei Medical University, Taipei 11696, Taiwan, R.O.C.
\end{abstract}

Received October 12, 2012; Accepted January 3, 2013

DOI: $10.3892 / \mathrm{ol} .2013 .1211$

\begin{abstract}
Mefloquine (MQ) is a prophylactic anti-malarial drug. Previous studies have shown that MQ induces oxidative stress in vitro. Evidence indicates that reactive oxygen species (ROS) may be used as a therapeutic modality to kill cancer cells. This study investigated whether MQ also inhibits prostate cancer $(\mathrm{PCa})$ cell growth. We used sulforhodamine $\mathrm{B}$ (SRB) staining to determine cell viability. MQ has a highly selective cytotoxicity that inhibits PCa cell growth. The antitumor effect was most significant when examined using a colony formation assay. MQ also induces hyperpolarization of the mitochondrial membrane potential (MMP), as well as ROS generation. The blockade of MQ-induced anticancer effects by $\mathrm{N}$-acetyl cysteine (NAC) pre-treatment confirmed the role of ROS. This indicates that the MQ-induced anticancer effects are caused primarily by increased ROS generation. Moreover, we observed that MQ-mediated ROS simultaneously downregulated Akt phosphorylation and activated extracellular signal-regulated kinase (ERK), c-Jun $\mathrm{N}$-terminal kinase
\end{abstract}

Correspondence to: Dr Liang-Ming Lee, Department of Urology, Wan Fang Hospital, Taipei Medical University, 111 Sec. 3 Xinglong Road, Taipei 11696, Taiwan, R.O.C.

E-mail:1m@tmu.edu.tw

${ }^{*}$ Contributed equally

Abbreviations: MQ, mefloquine; PCa, prostate cancer; ROS, reactive oxygen species; MMP, mitochondrial membrane potential; $\mathrm{NAC}, \mathrm{N}$-acetyl cysteine

Key words: mefloquine, prostate cancer, reactive oxygen species, mitochondrial membrane potential, hyperpolarization, $\mathrm{N}$-acetyl cysteine
(JNK) and adenosine monophosphate-activated protein kinase (AMPK) signaling in PC3 cells. These findings provide insights for further anticancer therapeutic options.

\section{Introduction}

Prostate cancer $(\mathrm{PCa})$ is the most frequently diagnosed malignancy in males and a common cause of cancer-related mortality. Hormone deprivation is an option for rapid androgen ablation in PCa patients. However, cancer cells eventually recur and progress to the androgen-independent stage. The treatments available for patients during this stage are limited.

Chloroquine (CQ) is a traditional anti-malarial medication that has been identified as a potential adjuvant in the treatment regimen of glioblastoma multiforme (GBM) (1). The results of clinical trials $(2,3)$ indicate that CQ is a potential adjuvant therapy for glioblastoma. Moreover, mefloquine (MQ) has been shown to be more potent than CQ in killing cancer cells in vitro and is potentially more efficacious than $\mathrm{CQ}$ as a chemotherapeutic agent for GBM patients (1). MQ is a valuable anti-malarial drug for prophylaxis and treatment for the majority of patients (4). MQ plasma concentrations reached $1,692 \mathrm{ng} / \mathrm{ml}(4.48 \mu \mathrm{M})$ for chemosuppression in Plasmodium falciparum infections $(5,6)$. Krudsood et al $(7)$ reported that an MQ plasma concentration of 5,796 $\mathrm{ng} / \mathrm{ml}(15.35 \mu \mathrm{M})$ was attained in a clinical study on P. falciparum-infected adults. Children tolerate MQ better than adults, and males tolerate it better than females (8).

This study examined two PCa cell lines (DU145 and PC3). DU145 and PC3 are the most commonly used PCa cell lines and their characteristics differ. PC3 was isolated from a bone metastasis, whereas DU145 cells were isolated from the brain metastases of prostate carcinoma. However, p53-mutant DU145 $(9,10)$ and p53-null PC3 $(9,11)$ cells are androgen-independent and proliferate normally in androgen-deprived media. 
This study had the following two objectives: i) to determine whether MQ possesses anticancer effects at potential therapeutic concentrations in vitro; and ii) to detail general MQ features in the context of disrupted cancer cell proliferation. This study shows that MQ exposure causes immediate hyperpolarization of mitochondrial membrane potential (MMP) and increases reactive oxygen species (ROS) generation in PCa cells. For the first time, the study also shows that MQ-mediated ROS inhibits Akt phosphorylation and activates c-Jun N-terminal kinase (JNK), extracellular signal-regulated kinase (ERK) and adenosine monophosphate-activated protein kinase (AMPK) signaling.

\section{Materials and methods}

Cell culture. Human foreskin fibroblast Hs68 cells and the androgen-independent PCa cell lines, PC3 and DU145, were maintained in Dulbecco's modified Eagle's medium and supplemented with $10 \%$ fetal bovine serum. The PCa cells were continuously cultured in a regular cell culture medium with $2 \mathrm{mM} \mathrm{L}$-glutamine, $100 \mu \mathrm{g} / \mathrm{ml}$ streptomycin and $100 \mathrm{U} / \mathrm{ml}$ penicillin in a humidified $5 \% \mathrm{CO}_{2}$ atmosphere. This study was approved by the Taipei Medical University Wan Fang Hospital.

Cell viability assay. Cells were seeded onto 96-well plates at a density of 5,000 cells per well and incubated for 1 day. Cell viability was assayed using sulforhodamine B (SRB) staining, as described previously (12). Absorbance at $570 \mathrm{~nm}$ was measured using an ELISA reader. Cell viability is expressed as the percentage of absorbance of the treated cells relative to that of the untreated (control) cells.

Colony formation assay. One hundred cells were seeded in a $10-\mathrm{cm}$ culture dish for $24 \mathrm{~h}$ and then incubated with MQ (or without for control). Colonies $>0.5 \mathrm{~mm}$ were counted after 2 weeks. Colonies were washed with phosphate-buffered saline (PBS) then air-dried, stained with $0.4 \%$ crystal violet for $1 \mathrm{~min}$, rinsed in water, air-dried and then photographed.

MMP assessment. MMP was measured using $40 \mu \mathrm{M}$ cationic lipophilic fluorochrome DiOC6. Cells were treated with MQ $\left(5 \times 10^{5}\right.$ cells/well) in a 6 -well plate for $40 \mathrm{~min}$, then DiOC6 was added and the cells were cultured continuously without light for $20 \mathrm{~min}$ at $37^{\circ} \mathrm{C}$ in the presence of MQ. Cells were then obtained and washed with $1 \mathrm{ml}$ ice-cold PBS. Finally, the cells were suspended in PBS and analyzed immediately using flow cytometry (FC500, Beckman Coulter, Miami, FL, USA). DiOC6 was recorded by fluorescence. The mean value of the MMP in the treated cells was calculated for comparison with that of the control cells.

Intracellular ROS assays. A 5- $\mu \mathrm{M}$ non-fluorescent 2',7'-dichlorofluorescein-diacetate (DCFH-DA) intracellular probe was used to detect ROS formation. Cells were treated with MQ $\left(5 \times 10^{5}\right.$ cells/well) in a 6-well plate for $40 \mathrm{~min}$, then DCFH-DA was added and the cells were cultured continuously without light for $20 \mathrm{~min}$ at $37^{\circ} \mathrm{C}$. Finally, cells were collected by centrifuging and suspended in $1 \mathrm{ml}$ ice-cold PBS. The oxidation of DCFH by ROS was determined by measuring the mean fluorescent intensity of DCFH by flow cytometry (FC500).
Western blot analysis. PC3 cells were pre-treated with $10 \mathrm{mM}$ $\mathrm{NAC}$ for $20 \mathrm{~min}$ and then treated with $10 \mu \mathrm{M}$ MQ for $1 \mathrm{~h}$. These were compared to the control cells which were treated with $10 \mu \mathrm{M}$ MQ for $1 \mathrm{~h}$ only. At harvest, total protein extracts were prepared and the protein concentration was determined using the Bradford method. Aliquots containing $20 \mu \mathrm{g}$ total protein each were subjected to western blot analysis. Antibodies against glyceraldehyde-3-phosphate dehydrogenase were purchased from Santa Cruz Biotechnology (Santa Cruz, CA, USA). Antibodies against phosphorylated Akt, JNK, AMPK and ERK were purchased from Cell Signaling Technology (Danvers, MA, USA).

Statistical analysis. Error bars represent the standard error of means (SEM) from independent triplicates $(n=3)$. All data are expressed as mean \pm SEM. We employed Sigma Plot 2001 software for statistical analysis. $\mathrm{P}<0.05$ was considered to indicate a statistically significant result.

\section{Results}

Effects of MQ on the proliferation of PCa and Hs68 cells. Two established PCa cell lines (DU145 and PC3) were examined for their sensitivity to MQ in vitro. We examined the growth inhibitory effects of MQ on PCa and Hs68 cells using SRB staining (Fig. 1). The PCa cell population was reduced after $24 \mathrm{~h}$ exposure to MQ ( $\mathrm{IC}_{50} \sim 10 \mu \mathrm{M}$ MQ, Fig. $1 \mathrm{~A}$ and B). PCa cell proliferation was completely inhibited at MQ concentrations $>20 \mu \mathrm{M}$. Assessment of Hs68 cells following MQ treatment showed no reduction in cell viability at $10 \mu \mathrm{M}$ (Fig. 1C). Treatment of the Hs68 cells with MQ resulted in an effect of $<\mathrm{IC}_{10}$ at $\sim 20 \mu \mathrm{M}$ MQ at $24 \mathrm{~h}$. Hs68 cells exhibited greater resistance to $\mathrm{MQ}$ exposure compared with the $\mathrm{PCa}$ cells. The results show that the $\mathrm{IC}_{50}$ value of MQ for PCa cells was $\sim 10 \mu \mathrm{M}$. MQ is a highly cytotoxic drug for PCa cell lines and causes $\sim 50 \%$ cell death in DU145 and PC3 at clinically achievable concentrations. Despite its anticancer potency, MQ is relatively nontoxic towards normal human cells.

Effects of $M Q$ on colony formation in PCa cells. A colony formation assay was performed to further show the antitumor effect of MQ (Fig. 2). Images of colonies grown in the presence or absence of MQ are shown in Fig. 2A (top, DU145; bottom, PC3). A significant antitumor effect was observed for MQ against the DU145 and PC3 cells (Fig. 2B).

Effects of MQ on MMP and ROS in PCa cells. MMP represents the performance of the electron transport chain and may indicate a pathological disorder of that system. A failure of mitochondrial bioenergetics is closely associated with the onset of apoptosis and necrosis. Since MQ is closely related to the alteration of MMP (13), this study investigated the effects of MQ on MMP using DiOC6. Following $1 \mathrm{~h}$ exposure to MQ $(10 \mu \mathrm{M})$, the fluorescence mean value of the MMP was $\sim 1.5$ - to 2-fold higher than that of the control PCa cells (Fig. 3A). Since MQ has a rapid effect on the hyperpolarization of MMP, the level of ROS produced within $1 \mathrm{~h}$ was further analyzed. The intracellular ROS levels in the MQ-treated PCa cells were assessed (Fig. 3B). PCa cells have a lower mean DCFH value in the absence of MQ than in its presence. MQ significantly 

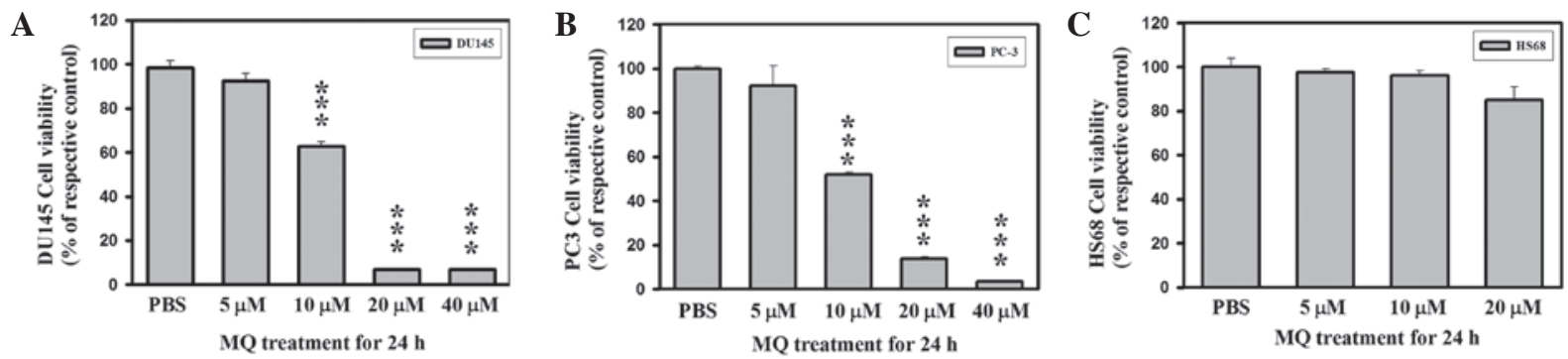

Figure 1. Inhibition of the proliferation of prostate cancer (PCa) cells by mefloquine (MQ) in vitro. (A) DU145, (B) PC3 and (C) Hs68 cells were treated with the indicated concentrations of MQ or phosphate-buffered saline (PBS; control) for $24 \mathrm{~h}$, and then assayed using sulforhodamine B (SRB) staining. Relative cell viability (\% of PBS control) is expressed as the mean \pm standard error of the mean (SEM). Error bars show the SEM $(\mathrm{n}=3) .{ }^{* * * *} \mathrm{P}<0.001$.
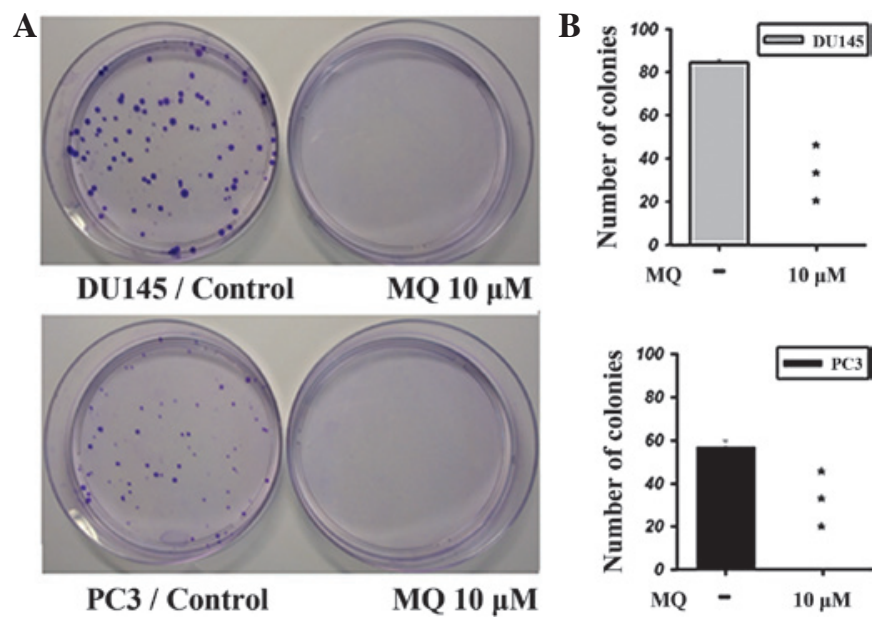

Figure 2. Antitumor effect of mefloquine (MQ) in DU145 and PC3 cells was assayed by colony formation assay. (A) Colony images and (B) relative number of colonies. Error bars show the standard error of the mean $(\mathrm{SEM} ; \mathrm{n}=3){ }^{* * * *} \mathrm{P}<0.001$ vs. the control.

A

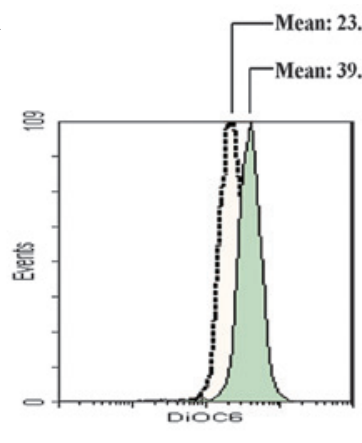

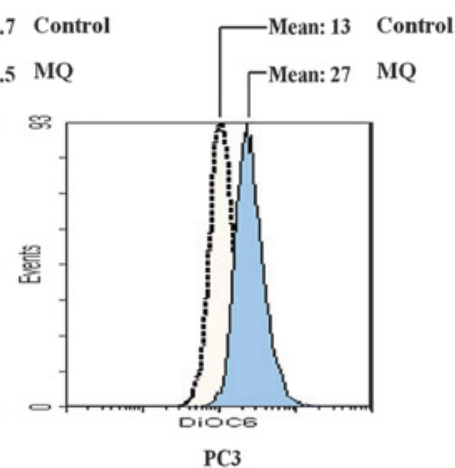

B

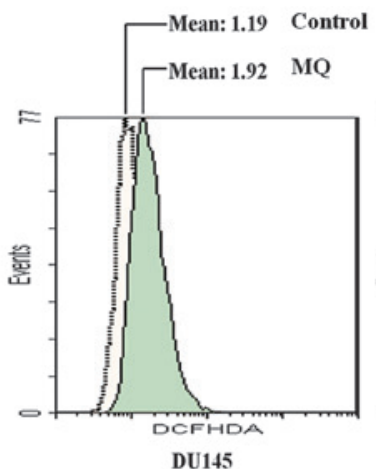

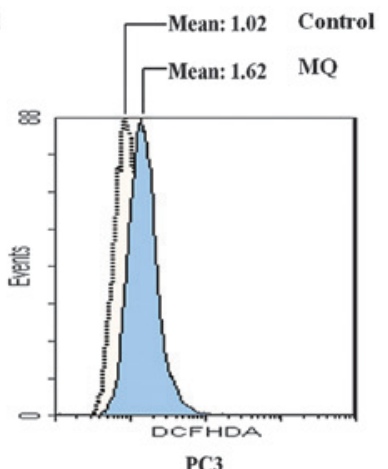

Figure 3. Effect of mefloquine (MQ)-induced alteration of mitochondrial membrane potential (MMP) and reactive oxygen species (ROS) generation in PCa cells. (A) DU145 and PC3 cells were treated with MQ (10 $\mu \mathrm{M})$ for $60 \mathrm{~min}$, and the change in MMP was analyzed by flow cytometry with DiOC6. (B) DU145 and PC 3 cells were treated with the indicated concentration of MQ $(10 \mu \mathrm{M})$ for $1 \mathrm{~h}$, and the ROS level was determined using a 2',7'-dichlorofluorescein-diacetate (DCFH-DA) dye.

stimulated the generation of ROS by PCa cells, as observed by the alteration of DCFH fluorescence. When treated with $10 \mu \mathrm{M}$ $\mathrm{MQ}$, the DCFH fluorescence increased from 1.19 to 1.92 and from 1.02 to 1.62 in DU145 and PC3 cells, respectively.

Pre-treatment with N-acetyl cysteine (NAC) protects DU145 and PC3 cells against MQ-induced anticancer effects and ROS-mediated signaling. Previous studies have suggested that preincubation with NAC is required to inhibit the cytotoxicity of generated ROS (14-16). NAC, which contains a sulfhydryl group, acts as a ROS scavenger and a precursor of intracellular reduced glutathione to regulate the redox status in the cells. To confirm the role of ROS in MQ-induced anticancer effects, cell viability was detected in DU145 and PC3 cells pre-treated with NAC for 20 min and then treated with $10 \mu \mathrm{M}$ MQ for $24 \mathrm{~h}$. Cell viability was determined using SRB staining. NAC pre-treatment significantly inhibited MQ-induced anticancer effects in PC3 and DU145 cells (Fig. 4A and B). The NAC 

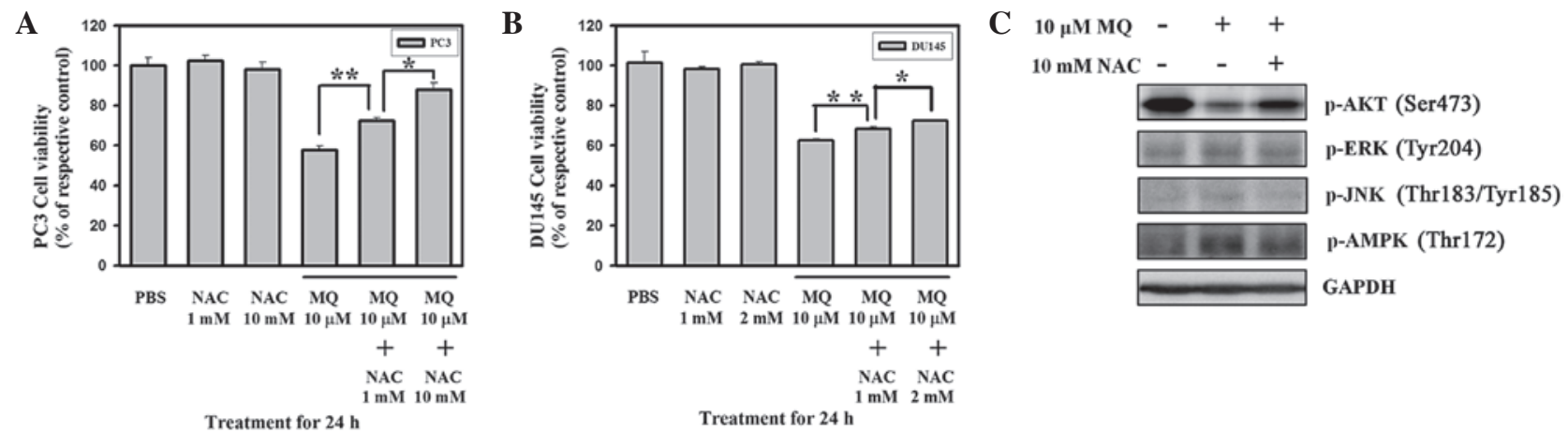

Figure 4. Pre-treatment with N-acetyl cysteine (NAC) disrupts mefloquine (MQ)-induced anticancer effects through reactive oxygen species (ROS) scavenging. (A) PC3 and (B) DU145 cells were pre-treated with the indicated NAC concentrations for 20 min before incubation with MQ (10 $\mu \mathrm{M})$ for $24 \mathrm{~h}$ and subsequent monitoring of cell viability. Relative cell viability [\% of phosphate-buffered saline (PBS) control] is expressed as the mean \pm standard error of the mean (SEM). Error bars show SEM $(\mathrm{n}=3)$. "P<0.05; ${ }^{* * *} \mathrm{P}<0.01$. (C) MQ-mediated signal transduction involving ROS-induced signaling alteration was also assayed. PC 3 cells were seeded in 6-well plates for $24 \mathrm{~h}$ and then drug-treated for $1 \mathrm{~h}$. Cell lysates were analyzed by western blot analysis for p-Akt, p-JNK, p-ERK and p-AMPK. JNK, c-Jun N-terminal kinase; ERK, extracellular signal-regulated kinase; AMPK, adenosine monophosphate-activated protein kinase.

control groups (PC3, 1 and $10 \mathrm{mM}$; DU145, 1 and $2 \mathrm{mM}$ ) showed no significant changes in cell viability compared with the untreated controls. These results indicate that MQ-mediated cytotoxicity is quenched by the antioxidant NAC. These data may also indicate that increased ROS generation is essential in MQ-mediated cell death. MQ-mediated signal transduction pathways involving ROS-induced signaling modulation were also investigated (Fig. 4C). We observed that MQ-mediated ROS simultaneously downregulated Akt Ser473 phosphorylation and activated ERK, JNK and AMPK signaling in PC3 cells. Moreover, Akt signaling was rescued and phosphorylation of ERK Tyr204, JNK Thr183/Tyr185 and AMPK Thr172 was decreased by pre-treatment with NAC, the ROS scavenger.

\section{Discussion}

MQ is a prophylactic anti-malarial drug that has been shown to possess antineoplastic activity against an experimental GBM tumor (1). Dow et al (17) showed that higher MQ blood levels are achieved under therapeutic regimens $(2.1$ to $23 \mu \mathrm{M})$ than under prophylaxis $(3.8 \mu \mathrm{M})$. This study was designed to understand the efficacy underlying MQ-induced anticancer effects. Human PCa cell lines, including commonly used androgen-independent DU145 and PC3 (18), were used.

Exposure to $10 \mu \mathrm{M}$ MQ for $24 \mathrm{~h}$ decreased the populations of DU145 and PC3 cells as determined by SRB staining (Fig. 1). The clonogenic effect was most significantly diminished when examined using a colony formation assay (Fig. 2). However, a normal human skin fibroblast cell line (Hs68) exhibited a different response to MQ exposure. Hs68 cells continued to proliferate normally in the presence of 10-20 $\mu \mathrm{MMQ}$, whereas the cancer cells were sensitive to the cytotoxic effects of MQ at $10 \mu \mathrm{M}$. This shows that MQ has highly selective anticancer capabilities.

Mitochondria are required as the primary source of ROS in cells. Mitochondrial dysfunction, oxidative stress and impaired cerebral energy metabolism contribute to cell death (19). The mitochondria are the primary organelles for production of high-energy phosphate. MMP hyperpolarization has also been associated with subcellular necrosis (20). In cell suicide (apoptosis and necrosis), mitochondrial hyperpolarization is required for specific steps of cell death (21). Previous studies have indicated that MQ treatment induces the cytotoxic effect that is dependent on the increase in oxidative stress in primary rat cortical neurons (22). MMP hyperpolarization may also increase ROS production (23). Exogenous oxidative stress may induce transient hyperpolarization of MMP and a delayed burst of endogenous ROS (24). MMP hyperpolarization and ROS generation are early molecular events that precede cell death (25). In certain types of necrosis, cell death is mediated by an increase in the MMP-dependent overproduction of ROS (21). A recent study (26) has shown that massive mitoptosis may result in cell death. ROS-initiated mitoptosis is presumed to eliminate the mitochondria which overproduce ROS (21). MMP hyperpolarization-induced ROS cause oxidative stress-induced necrotic cell death (24).

MMP alteration and oxidative stress in cancer cells treated with MQ have not been examined previously. The current study investigated MMP alteration induced by $1 \mathrm{~h}$ of exposure to MQ in PCa cells. In the alteration of MMP, a change in the state of ROS was also observed. In our study, MQ treatment led to significant MMP hyperpolarization. This indicates that MMP hyperpolarization during MQ treatment mediated intracellular ROS generation which inhibited PCa cells at concentrations $>10 \mu \mathrm{M}$.

There are several signaling factors, such as Akt, AMPK and the mitogen-activated protein kinase (MAPK) family, that regulate the progression of various tumors. Akt is activated by Ser473 phosphorylation, which affects cell growth, proliferation and survival. The increased ROS levels may enhance MAPK activities. The phosphorylation of AMPK Thr172 depends on cellular metabolic stress. Previous studies have implied that the signaling factors Akt, AMPK and the MAPK family are potentially associated with ROS-triggered biological responses. ROS are a significant type of molecule that mediate numerous signal transduction pathways and have a critical function in cells. To confirm the function of ROS generation in MQ treatment, NAC was used to scavenge the ROS. Pre-treatment of DU145 and PC3 cells with NAC abrogated ROS induction by MQ and significantly protected these cells against MQ-induced anticancer effects. Exposure of PC3 cells to MQ for $1 \mathrm{~h}$ caused a decrease in phosphoryla- 
tion of Akt at Ser473. Simultaneously, we also observed the activation of AMPK (at Thr172) and MAPK family members (ERK at Tyr204 and JNK at Thr183/Tyr185) in response to MQ treatment. Pre-treatment with NAC confirmed the function of Akt, ERK, JNK and AMPK in MQ treatment. Blocking ROS generation with NAC had a significantly preventative effect on Akt activation, and inhibited ERK, JNK and AMPK. This study indicates an anticancer action of MQ that is dependent on Akt signaling disruption and JNK, ERK and AMPK signaling upregulation in PCa cells; this action is dependent on ROS generation. Mitochondrial function and ROS generation are involved in PI3K-Akt-mTOR and MAPK (JNK/ERK) signaling for autophagy in cancer (27). AMPK regulates the ROS/redox balance which indicates that AMPK signaling is critical in intracellular homoeostasis during autophagy (28). The intricate relationships between ROS-mediated signaling (involving Akt, JNK, ERK and AMPK) and autophagy require further study. We corroborate that MQ induces ROS generation which then affects cancer cell proliferation by modulating signaling transduction, specifically by ERK, JNK and AMPK activation and Akt inhibition.

Thus, we assert that MQ has a strong inhibitory effect on cell viability by producing ROS in DU145 and PC3 cells. These results indicate that MQ may be a potential candidate for clinical trials of cancer applications in the future.

\section{Acknowledgements}

This study was supported by the Research Fund of the Center of Excellence for Cancer Research, Taipei Medical University, Taipei, Taiwan (Project Number DOH100-TD-C-111-008) and the National ScienceCouncil(GrantNSC 100-2314-B-038-039).

\section{References}

1. Geng Y, Kohli L, Klocke BJ and Roth KA: Chloroquine-induced autophagic vacuole accumulation and cell death in glioma cells is p53 independent. Neuro Oncol 12: 473-481, 2010.

2. Sotelo J, Briceno E and López-González MA: Adding chloroquine to conventional treatment for glioblastoma multiforme: a randomized, double-blind, placebo-controlled trial. Ann Intern Med 144: 337-343, 2006.

3. Briceño E, Reyes S and Sotelo J: Therapy of glioblastoma multiforme improved by the antimutagenic chloroquine. Neurosurg Focus 14: e3, 2003.

4. Taylor WR and White NJ: Antimalarial drug toxicity: a review. Drug Saf 27: 25-61, 2004.

5. Simpson JA, Price R, ter Kuile F, Teja-Isavatharm P, Nosten F, Chongsuphajaisiddhi T, Looareesuwan S, Aarons L and White NJ: Population pharmacokinetics of mefloquine in patients with acute falciparum malaria. Clin Pharmacol Ther 66: 472-484, 1999

6. Kollaritsch H, Karbwang J, Wiedermann G, Mikolasek A, Na-Bangchang K and Wernsdorfer WH: Mefloquine concentration profiles during prophylactic dose regimens. Wien Klin Wochenschr 112: 441-447, 2000.

7. Krudsood S, Looareesuwan S, Wilairatama P, Leowattana W, Tangpukdee N, Chalermrut K, Ramanathan S, Navaratnam V, Olliaro P, Vaillant M, Kiechel JR and Taylor WR: Effect of artesunate and mefloquine in combination on the Fridericia corrected QT intervals in Plasmodium falciparum infected adults from Thailand. Trop Med Int Health 16: 458-465, 2011.

8. ter Kuile FO, Nosten F, Thieren M, Luxemburger C, Edstein MD, Chongsuphajaisiddhi T, Phaipun L, Webster HK and White NJ: High-dose mefloquine in the treatment of multidrug-resistant falciparum malaria. J Infect Dis 166: 1393-1400, 1992.
9. Isaacs WB, Carter BS and Ewing CM: Wild-type p53 suppresses growth of human prostate cancer cells containing mutant p53 alleles. Cancer Res 51: 4716-4720, 1991.

10. Gurova KV, Rokhlin OW, Budanov AV, Burdelya LG, Chumakov PM, Cohen MB and Gudkov AV: Cooperation of two mutant p53 alleles contributes to Fas resistance of prostate carcinoma cells. Cancer Res 63: 2905-2912, 2003.

11. Bajgelman MC and Strauss BE: The DU145 human prostate carcinoma cell line harbors a temperature-sensitive allele of p53. Prostate 66: 1455-1462, 2006.

12. Keepers YP, Pizao PE, Peters GJ, van Ark-Otte J, Winograd B and Pinedo HM: Comparison of the sulforhodamine B protein and tetrazolium (MTT) assays for in vitro chemosensitivity testing. Eur J Cancer 27: 897-900, 1991.

13. McArdle JJ, Sellin LC, Coakley KM, Potian JG and Hognason K: Mefloquine selectively increases asynchronous acetylcholine release from motor nerve terminals. Neuropharmacology 50: 345-353, 2006.

14. Rogalska A,Koceva-Chyla A and JóźwiakZ: Aclarubicin-induced ROS generation and collapse of mitochondrial membrane potential in human cancer cell lines. Chem Biol Interact 176: 58-70, 2008.

15. Liu H, Jiang C, Xiong C and Ruan J: DEDC, a new flavonoid induces apoptosis via a ROS-dependent mechanism in human neuroblastoma SH-SY5Y cells. Toxicol In Vitro: 26: 16-23, 2012.

16. Lan A, Liao X, Mo L, Yang C, Yang Z, Wang X, Hu F, Chen P, Feng J, Zheng D and Xiao L: Hydrogen sulfide protects against chemical hypoxia-induced injury by inhibiting ROS-activated ERK1/2 and p38MAPK signaling pathways in PC12 cells. PLoS One 6: e25921, 2011.

17. Dow GS, Caridha D, Goldberg M, Wolf L, Koenig ML, Yourick DL and Wang Z: Transcriptional profiling of mefloquine-induced disruption of calcium homeostasis in neurons in vitro. Genomics 86: 539-550, 2005.

18. Chlenski A, Nakashiro K, Ketels KV, Korovaitseva GI and Oyasu R: Androgen receptor expression in androgen-independent prostate cancer cell lines. Prostate 47: 66-75, 2001.

19. Robertson CL, Scafidi S, McKenna MC and Fiskum G: Mitochondrial mechanisms of cell death and neuroprotection in pediatric ischemic and traumatic brain injury. Exp Neurol 218: 371-380, 2009.

20. Berghe TV, Vanlangenakker N, Parthoens E, Deckers W, Devos M, Festjens N, Guerin CJ, Brunk UT, Declercq W and Vandenabeele P: Necroptosis, necrosis and secondary necrosis converge on similar cellular disintegration features. Cell Death Differ 17: 922-930, 2010.

21. Skulachev VP: Bioenergetic aspects of apoptosis, necrosis and mitoptosis. Apoptosis 11: 473-485, 2006.

22. Hood JE, Jenkins JW, Milatovic D, Rongzhu L and Aschner M: Mefloquine induces oxidative stress and neurodegeneration in primary rat cortical neurons. Neurotoxicology 31: 518-523, 2010.

23. Zheng Z, Chen H, Zhao H, Liu K, Luo D, Chen Y, Yang X, $\mathrm{Gu} \mathrm{Q}$ and $\mathrm{Xu} \mathrm{X}$ : Inhibition of JAK2/STAT3-mediated VEGF upregulation under high glucose conditions by PEDF through a mitochondrial ROS pathway in vitro. Invest Ophthalmol Vis Sci 51: 64-71, 2010.

24. Choi K, Kim J, Kim GW and Choi C: Oxidative stress-induced necrotic cell death via mitochondria-dependent burst of reactive oxygen species. Curr Neurovasc Res 6: 213-222, 2009.

25. Di Stefano A, Frosali S, Leonini A, Ettorre A, Priora R, Di Simplicio FC and Di Simplicio P: GSH depletion, protein S-glutathionylation and mitochondrial transmembrane potential hyperpolarization are early events in initiation of cell death induced by a mixture of isothiazolinones in HL60 cells. Biochim Biophys Acta 1763: 214-225, 2006.

26. Venditti P, Di Stefano L and Di Meo S: Mitochondrial metabolism of reactive oxygen species. Mitochondrion: Jan 29, 2013 (Epub ahead of print)

27. Li ZY, Yang Y, Ming M and Liu B: Mitochondrial ROS generation for regulation of autophagic pathways in cancer. Biochem Biophys Res Commun 414: 5-8, 2011.

28. Wang S, Song P and Zou MH: AMP-activated protein kinase, stress responses and cardiovascular diseases. Clin Sci (Lond) 122: $555-573,2012$. 